\title{
Erratum to: Integrable Systems of Neumann Type
}

\author{
Alina Dobrogowska ${ }^{1,2}$. Tudor S. Ratiu ${ }^{3}$
}

Published online: 22 June 2016

C Springer Science+Business Media New York 2016

\section{Erratum to: J Dyn Diff Equat (2015) 27:533-553 DOI 10.1007/s10884-013-9314-5}

In Subsects. 3.2 and 3.3, pages 541-543, it is implicitly assumed that $\epsilon \neq 0$. This hypothesis should have been explicitly stated. Similarly, on page 552, in the sentence above Theorem 4.2, the hypothesis should be $\epsilon \in \mathbb{R} \backslash\{0\}$. The case $\epsilon=0$ is not considered in the paper; it was carried out, by hand, only for the system in Example 3.1, pages 544-545.

The online version of the original article can be found under doi:10.1007/s10884-013-9314-5.

$\triangle$ Tudor S. Ratiu

tudor.ratiu@epfl.ch

Alina Dobrogowska

alaryzko@alpha.uwb.edu.pl

1 Section de Mathématiques, École Polytechnique Fédérale de Lausanne, 1015 Lausanne, Switzerland

2 Institute of Mathematics, University of Białystok, Lipowa 41, 15-424 Bialystok, Poland

3 Section de Mathématiques and Bernoulli Center, École Polytechnique Fédérale de Lausanne, 1015 Lausanne, Switzerland 